\title{
Health Query Expansion Based on Graph Matching between DBpedia and UMLS
}

\author{
https://doi.org/10.3991/ijoe.v17i06.22755 \\ Sarah Dahir ${ }^{(\varpi)}$ \\ Moulay Ismail University Meknes, Morocco \\ sarah.dahir2012@gmail.com \\ Abderrahim El Qadi \\ Mohammed V University, Rabat, Morocco \\ Hamid Bennis \\ Moulay Ismail University, Meknes, Morocco
}

\begin{abstract}
Information Retrieval (IR) in the medical domain is considered as a challenging task for many reasons. Short health queries tend to lack information on user's intent, and the target corpus may not have sufficient information for Relevance Feedbacks. And even, if the user obtains relevant documents to his/her queries, it is difficult for him/her to understand the technical terms. In contrast, in this paper, we propose an approach for health queries reformulation based on graph matching between two external linked data sources: DBpedia and Unified Medical Language System (UMLS). DBpedia has a broad coverage of topics and less noise compared to Wikipedia articles, and UMLS is specific to the medical domain. We also introduced the degree centrality to measure the graph connectivity and to select the most efficient candidate terms for query expansion. Experimental results on MEDLINE collection using Okapi BM25 as a retrieval model showed that our approach outperformed related methods, and the two sources achieved very good retrieval results. They helped in the diversification of the retrieved documents and the improvement of the recall.
\end{abstract}

Keywords - Information Retrieval, Search Result Diversification, Query Reformulation, Linked data, Graph matching, Degree centrality

\section{Introduction}

Nowadays, health related information is increasingly available through several biomedical data sources including clinical reports and forums among others. Moreover, a survey indicates that around $80 \%$ population of US search engine users look for information on particular diseases or health problems [1].

This increasing demand for domain-specific IR from medical practitioners has led communities like TREC and CLEF to gather health resources and foster research in this field [2]. One of largest well-known databases of biomedical literatures is 
MEDLINE. This database is freely accessible via the Web interface PUBMED, which contains over 26 million references to medical documents [3], and about 500,000 new citations are added to it on an annual basis [2].

However, it can be extremely difficult to correspond the expressions used in health queries to domain experts' ones. And even if the user obtains relevant results to his/her queries, it is difficult for him/her to understand the technical terms from the medical domain especially that abbreviations are often used in the biomedical literature [4]. Luckily, a first initiative, called Consumer Health Vocabulary (CHV) [5] took place to solve this vocabulary gap problem by translating the technical terms from the health domain to consumer-friendly ones. The CHV was later added to the well-known ontology UMLS that aims at enabling the interoperability between computer systems by bringing together several health and biomedical vocabularies and standards [6].

UMLS is a domain-specific ontology, it partially solves the problem of multivalued features which is a common problem for the non-domain-specific linked data sources. In UMLS; concepts are identified by Concept Unique Identifiers (CUI) that contain the letter $\mathrm{C}$ followed by seven numbers e.g. C0023317. Also, UMLS allows the recognition of concepts in a text, of maximum 10,000 characters in a single block, thanks to MetaMap1 which maps biomedical text to the UMLS Metathesaurus 2 . However, its search engine returns ranked results with restrictions like the search string cannot exceed 75 characters. Also, there are not many semantic relations in UMLS. For instance, there are only 15 semantic types in the 2019AA3 release of UMLS, including, among others, the "empty relationship" and the "has child relationship" ("CHD"). And in terms of granularity; the Metathesaurus of UMLS comprises over 2 million names for 900,000 biomedical concepts from more than 60 families of biomedical vocabularies like the Medical Subject Headings (MeSH) [6].

However, ontology-based methods have many issues such as the vocabulary mismatch problem between the query terms and the concepts from the ontology, and the difficulty to construct a domain-dependent ontology from scratch [7].

In this work, we integrate DBpedia knowledge base domain-ontology to enhance the performance of UMLS ontology in the medical domain. We reformulate the health-related queries using the graph matching between UMLS and DBpedia. In fact, DBpedia has a large variety of semantic features. And as for its granularity, the 201604version of DBpedia describes 6.0 million things, out of which 5.2 million are classified in a constant ontology including 5,000 diseases [8]. Also, we introduce the degree centrality results to select the efficient terms for expanding the initial user query. And by diversifying query expansion, we allow Search Result Diversification (SRD) [9]. Consequently, the user is more likely to find relevant results to his/her query in the top retrieved documents since each of them will be covering a different aspect of the query.

${ }^{1}$ https://metamap.nlm.nih.gov/

${ }^{2}$ https://uts.nlm.nih.gov/metathesaurus.html

${ }^{3}$ https://www.nlm.nih.gov/research/umls/knowledge_sources/metathesaurus/release/abbreviations.html 
This paper is organized as follows Section 2 discusses related works. Section 3 depicts our approaches, and section 4 addresses the obtained results, and gives an outlook on our future works.

\section{Related Works}

Recently, some studies suggested using external resources as well as graph matching to improve query expansion. Query reformulation through the combination of multiple information sources has a better effectiveness compared to the use a single information source [10]. Diverse sources such as Wikipedia [11] were found to be beneficial for document retrieval.

In [12], authors performed query expansion on the MEDLINE collection by exploiting the top retrieved Wikipedia articles, and using their corresponding Wikidata attributes' values. However, even if Wikidata has links to UMLS as well as to other databases, using UMLS directly would have been more appropriate and a better alternative.

Other works [13], introduced external "document expansion" from Wikipedia through "document reduction" that generates a query for a document. This approach has a disadvantage; because in general a feedback document is independently relevant to the query, but a feedback document from Wikipedia may correspond only to a segment from the query and not to the whole of it [14]. Also, authors' study in [15] showed that top retrieved documents contain $65 \%$ harmful terms.

Another kind of studies [16] identified objects within a query and gave them ranking scores using Google Search API, then performed Pseudo Relevance Feedback (PRF) on linked objects' descriptions, from Freebase, to select the expansion terms. For instance, an object (entity) in Freebase is identified by a unique Machine id. Yet, the granularity of the Freebase ontology's first level can be too general for certain queries; and lower levels are difficult to use because of the lack of instances.

Most of the RDF graph matching algorithms are either involving a pairwise comparison of semantic resources like SimRank [17] that considers objects as similar if they are related to similar objects, or based on finding paths between resources like LDSD [18] which provides measures for the determination of the semantic distance between Linked Data resources. Moreover, such algorithms do not use the predicates that usually contain valuable information [19].

Also, using the whole graph representation of information sources to exploit their matching relationships; is highly demanding computationally [20]. To overcome these problems, authors in [20] explored for each WordNet concept, a WordNet sub-graph centering on it as well as a UMLS sub-graph of candidate matches to identify the matching relationships between the two ontologies. In our work we used DBpedia instead of WordNet; that exploits terms individually i.e., it does not take into account the context of the term to determine its meaning. Also, unlike DBpedia that we used in this work, WordNet covers only few relations which are synonymy, hypernymy, and hyponymy. Moreover, the WordNet concepts follow a tree structure whereas 
UMLS has a general graph structure [20]. As a consequence, UMLS has a better connectivity.

Based on RDF graph matching; authors in [19] enhanced user satisfaction by generating intelligent snippets (i.e. snippets providing more valuable information): First, they generated RDF graphs for queries and documents using WordNet (for expanding queries with synonyms) and DBpedia Spotlight (for named-entities recognition) among others. Second, they transformed the graphs to bipartite graphs [21]. Third, they opted either for the resource-graph matching algorithm: Relevance Search (RS) [22] that allows comparing a node with a graph, or for the graph-graph matching algorithm that determines the common resources in two graphs for the snippets generation.

In [7], authors used a WordNet graph-based method to expand queries by selecting all synonyms, hypernyms, etc. of each query term to obtain a sub-graph including only the shortest path between a pair of query terms. This method had liabilities because it used WordNet that may not have ontology for a certain domain. Moreover, WordNet has a low coverage of concepts and phrases [23] compared to DBpedia which annotates entities that can be phrases.

And in [24], authors expanded biomedical [25] queries using MeSH thesaurus. Then, they retrieved documents based on the similarity between those expanded queries and clusters of biomedical documents. Unlike our approaches, this approach does not take advantage of other resources within UMLS.

\section{Proposed Approach}

This paper aims at formulating a new form of query expansion by integrating and matching between two external resources: DBpedia and UMLS (Figure 1).

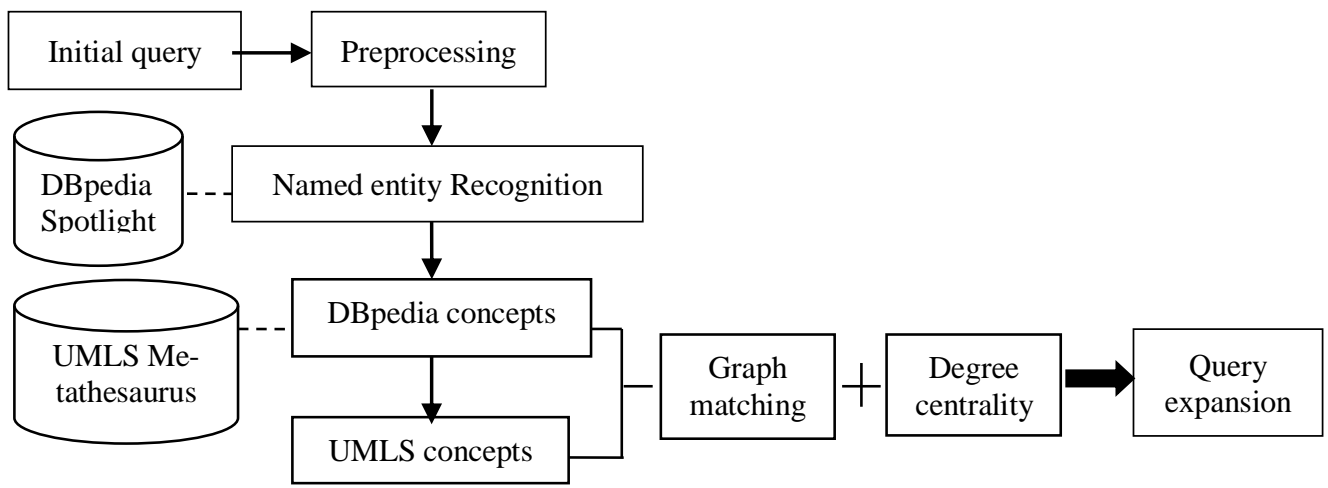

Fig. 1. The proposed IR process

Figure 1 describes our proposed method for query expansion that uses graph matching between DBpedia and UMLS ontologies as well as degree centrality. 
The process of query expansion is carried out according to the following phases:

1. Preprocessing of queries through stop-words' removal and stemming [26] using the Porter Stemmer.

2. Using DBpedia Spotlight to determine concepts (entities) in the query and divide its keywords to two categories concept/not concept (1).

$$
q_{I}=t_{c}+t_{n c}
$$

Where qi: the initial query,

$t_{c}$ :DBpedia concepts in the query, and $t_{n c}$ : non DBpedia concepts in the query.

3. Searching each of the found concepts in the UMLS Metathesaurus Browser.

4. Matching between DBpedia and UMLS: Matching directly related "dct:subject" values as well as indirectly related "skos:broader" and "is det:subject of" values of the DBpedia concept, with the attributes of the UMLS concept.

In fact, for a concept $\mathrm{i}$ in ontology $\mathrm{A}$, a concept $\mathrm{j}$ in ontology $\mathrm{B}$ is considered as a match of $i$ if $i$ and $j$ have similar meanings [20]. Similarly, in this work, we considered attributes from the two ontologies as matching ones, if they had either a relation of equivalence $(=)$ or if an attribute from the first ontology is more general $(\sqsupseteq)$ compared to the one from the other ontology as shown in figure 2. And we chose to use particularly the attributes "dct:subject", "is dct:subject of" and "skos:broader" from DBpedia for two reasons: first, in order to make our approach more simple and non expensive computationally. Second, we chose these attributes because they are commonly found for most entities and at the same time, we believe that they carry valuable information for the matching with UMLS.

In figure 2, we matched DBpedia concepts with UMLS ones. These UMLS concepts are connected to "aortic insufficiency" through the semantic types: "RB" which means "has a broader relationship" and "RO" which stands for: "has relationship other than synonymous, narrower, or broader". The "valvular heart desease" is a DBpedia category of the entity "aortic insufficiency" and it is directly linked to this entity. While "aortic stenosis" is actually an entity and "valvular heart disease" is one of its categories. That is to say, "aortic stenosis" is not directly linked to aortic insufficiency".

5. Reformulation of the initial query through:

Strategy 1: Using the candidates' terms that were commonly found in both of the external semantic sources (conceptsmatch of the 4th phase) as shown in (2): $\mathrm{q}_{\mathrm{E} 1}:(\text { DBpedia - UMLS })_{\text {GraphMatching }}$

$$
\mathrm{q}_{\mathrm{E} 1}=\mathrm{q}_{\mathrm{I}}+\text { concepts }_{\text {match }}
$$

Strategy 2: Using "degree centrality" measure to determine query reformulation terms from the ones obtained in the 4th step by using both the content of the nodes with degree centrality higher than the average, and the matching terms between their 
directly linked DBpedia nodes and the UMLS nodes as shown in (3). We examine the performance of the degree centrality:

$$
\mathrm{q}_{\mathrm{E} 2}:(\mathrm{DBpedia}-\mathrm{UMLS})_{\text {DegreeCentralityMatches }}
$$

$$
\mathrm{q}_{\mathrm{E} 2}=\mathrm{q}_{\mathrm{I}}+\mathrm{V}_{\text {DCaboveaverage }}+\text { degreecentrality }_{\text {matches }}
$$

Where VDCaboveaverage: the vertices having a Degree Centrality which is above the average;

And degreecentralitymatches: the conceptsmatch of the VDCaboveaverage' directly linked vertices i.e. matching terms between each directly linked DBpedia vertex to a VDCaboveaverage and an UMLS vertex.

In fact, the "degree centrality" measure is one of the well-known graph connectivity measures. It is a variant of "graph centrality" that aims at determining the importance of a node in a graph by considering the relation of the node with other nodes in the graph [23]. Actually, it is the simplest way to determine a vertex importance by its degree [27]. The degree of a vertex refers to the number of edges incident on that vertex [7]. And the degree centrality is the degree of a vertex normalized by the maximum degree [27].

Concept terms that are separated by "/" in UMLS are all used for query reformulation because they are considered as synonyms.

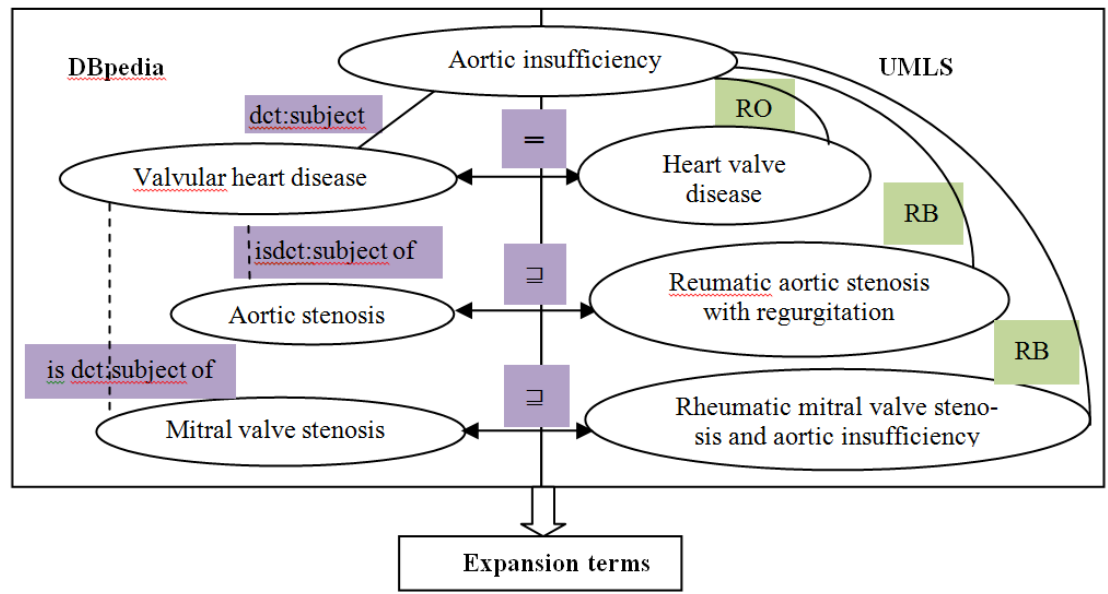

Fig. 2. A sub-graph of query 6 from MEDLINE collection that corresponds to the query terms "aortic regurgitation" (annotated as "aortic insufficiency" in DBpedia and "aortic valve insufficiency" in UMLS) 


\section{$4 \quad$ Experiments and Results}

\subsection{Dataset description}

To evaluate our approach, we used the MEDLINE collection, and Okapi BM25 as a retrieval model. We indexed this dataset using Indri4search engine.

The dataset's texts (table 1) vary in length and contain more technical terms.

Table 1. Description of the dataset

\begin{tabular}{|l|c|}
\hline \multicolumn{1}{|c|}{ Total number of texts } & $\mathbf{1 0 3 3}$ \\
\hline Number of topics & 30 \\
\hline Total number of tokens & 159970 \\
\hline Total number of distinct (unique) tokens & 13113 \\
\hline Average number of tokens per text & 100 \\
\hline
\end{tabular}

\subsection{Evaluation measures}

The overall performance was evaluated in terms of Recall $(\mathrm{R})$, Precision $(\mathrm{P})$, and MAP.

- Precision. Shows to which level a system is capable of returning only relevant documents [28]:

$$
\text { Precision }=\frac{\text { Number of relevant retrieved documents }}{\text { Number of retrieved documents }}
$$

- Recall. Is also called the true positive rate and it shows how capable a system is of returning all relevant documents [28]:

$$
\text { Recall }=\frac{\text { Number of relevant retrieved documents }}{\text { Number of relevant documents }}
$$

- MAP. The MAP for or a set of queries is the mean of the Average Precision (AP) scores for each query [29]:

$$
\operatorname{MAP}=\frac{\sum_{\mathrm{q}=1}^{\mathrm{Q}} \operatorname{AveP}(\mathrm{q})}{\mathrm{Q}}
$$

Where $\mathrm{Q}$ is the number of queries

\section{$5 \quad$ Results}

Table 2 represents the results obtained with our two suggested queries' reformulation methods. As well as their comparison with: the UMLS approach, that we per-

${ }^{4}$ https://www.lemurproject.org/indri/ 
formed using attributes that we obtained from step 3 of our method; prior to the matching step, and "Clusters' Retrieval Derived from Expanding Statistical Language Modeling Similarity and Thesaurus-Query Expansion with Thesaurus" (CRDESLMQET) [24] approach.

Table 2. Results of the comparison between our approaches and related approaches in terms of P@10 and R@10

\begin{tabular}{|l|c|c|}
\hline \multicolumn{1}{|c|}{ Approach } & P@ 10 & R @ 10 \\
\hline CRDESLM-QET [24] & 0,5 & 0,25 \\
\hline DBpedia & 0,666 & 0,643 \\
\hline UMLS & 0,705 & 0,616 \\
\hline (DBpedia-UMLS) $_{\text {GraphMatching }}$ & 0,707 & 0,595 \\
\hline (DBpedia-UMLS) $_{\text {DegreeCentralityMatching }}$ & 0,734 & 0,685 \\
\hline
\end{tabular}

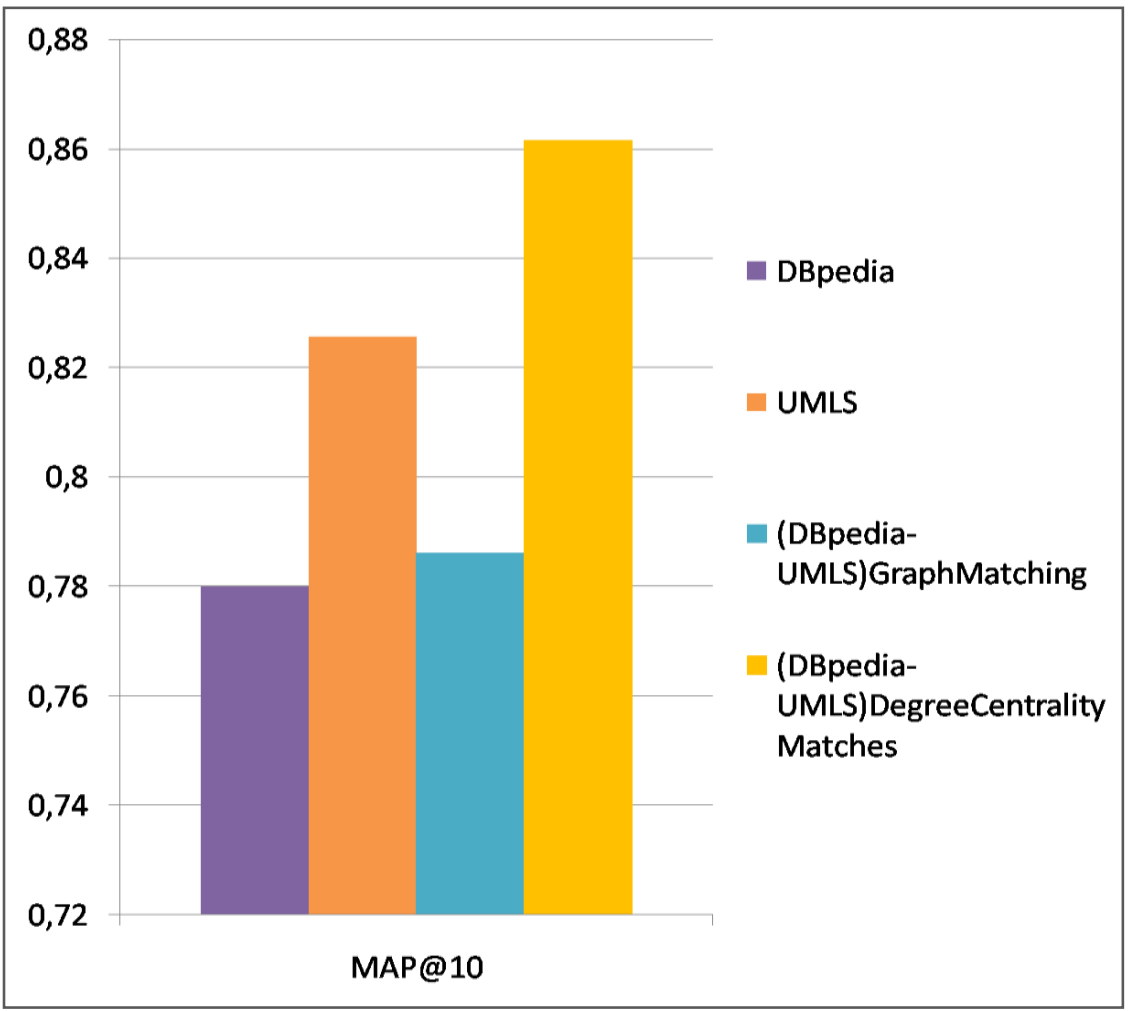

Fig. 3. MAP at 10 of our approaches

From the results in table 2 and figure 3, we notice that the (DBpedia-UMLS) degree centrality matches outperformed the (DBpedia-UMLS) graph matching for all used measures.

In both P@10 and R@10, our graph-based approaches outperformed considerably the CRDESLM-QET [24]. 
As for the use of every linked data source separately from the other (i.e. without matching them); the DBpedia approach gave lower precision and MAP at 10 compared to the (DBpedia-UMLS) graph matching. Whereas the UMLS approach gave a slightly better recall and MAP at 10 compared to the (DBpedia-UMLS) graph matching. And the P@10 of UMLS was nearly similar to that of our (DBpedia-UMLS) graph matching.

Also, the UMLS approach gave better results than the DBpedia approach in terms of P@10 and MAP@10. We believe that this difference is normal since UMLS is specialized in the medical domain. Thus, it is richer in terms of technical terms of that domain compared to DBpedia.

Moreover, we believe that our approaches boost significantly the recall because we are using linked data to expand queries. As a result, documents that do not contain initial queries' concepts but contain their interlinked concepts are retrieved. Consequently, non-domain expert users will be able to find relevant documents even when they are not knowledgeable about the domain to know the right terms to use. And even for experts, they can find other relevant documents they may be interested in since the expansion concepts are interlinked to their queries and cover more aspects of their intent. In other words, our approaches improve considerably the recall since we are adding different terms from different external resources and covering different aspects of the query concepts.

As for the graph matching approach, we think that the low results are due to the small number of features we used from both DBpedia and UMLS which already has a very low number of features. One way to improve the results of this first approach would be exploiting interlinked data of indirect features. In other words, we need to exploit concepts from the root to all their $n$ hop neighbors.

In this work, although the queries of the MEDLINE collection are very long and most of them contain two to three sentences, we opted for query expansion instead of query reduction which is another possibility for query reformulation. In fact, since we are dealing with a domain specific dataset and professional terms, almost every term in the query is mandatory for retrieving relevant documents; even if it is not recognized as a concept by DBpedia. In the future, we will find an accurate way to perform query reduction on such queries. Also, we will diversify even more the expansion terms by using multiple external sources. Another way to improve our approach is through: first, focusing on the matching between every source's objects in a separate way i.e. matching DBpedia objects within a query using their similar attributes. And second, matching attributes of a certain source like DBpedia with their equivalent attributes from other sources like we did in this work.

\section{Conclusion}

Related query expansion works tend to either combine linguistic features from a thesaurus like WordNet and semantic features from a non-domain specific linked data source, or rely only on one external source for graph matching. The novelty about our work is the application of graph matching as well as the use of degree centrality 
measure on two linked data sources; one of them is general and the other one is domain specific. Another advantage of our approach is covering and exploiting all query concepts and not only a certain segment or $\mathrm{n}$-gram of it as in related works that use Wikipedia feedback documents. And since we are using a graph matching method we do not process a concept separately from the others.

In this work, we performed query reformulation through: first, distinguishing between DBpedia concepts and non DBpedia concepts in the initial query. Second, the expansion of the query using external terms from DBpedia and UMLS based on degree centrality results as well as common matching terms. This approach lead to better recall results compared to related approaches. Consequently, we can say that reformulating queries [30] through matching between the graphs of external sources and using degree centrality measure helps; especially in the improvement of the recall because the expansion terms are more diverse and are based on semantics. As a result, multiplying the external information sources for query expansion; helps in the diversification of the retrieved documents and thus the improvement of the recall. In fact, external sources tend to expand the query from different aspects of its meaning rather than expanding it based only on the target document collection.

\section{$7 \quad$ References}

[1] Fox, S. (2011). Health topics: $80 \%$ of internet users look for health information online. Pew Internet \& American Life Project.

[2] Thakkar, H., Iyer, G., \&Majumder, P. (2015). A comparative study of approaches in usercentered health information retrieval. arXivpreprint arXiv:1505.01606.

[3] Wang, Y., Huang, L., Guo, S., Gong, L., \& Bai, T. (2019). A novel MEDLINE topic indexing method using image presentation. Journal of Visual Communication and Image Representation, 58, 130-137. https://doi.org/10.1016/j.jvcir.2018.11.022

[4] Le Maguer, S., Hamon, T., Grabar, N., \& Claveau, V. (2015, March). Recherche d'information médicale pour le patient Impact de ressources terminologiques. In COnférence en Recherche d'Information et Applications, CORIA 2015.

[5] Zeng, Q. T., \& Tse, T. (2006). Exploring and developing consumer health vocabularies. Journal of the American Medical Informatics Association, 13(1), 24-29.

[6] Bodenreider, O. (2004). The unified medical language system (UMLS): integrating biomedical terminology. Nucleic acids research, 32(suppl_1), D267-D270. https://doi.org/10. $\underline{\text { 1093/nar/gkh061 }}$

[7] Jain, A., Mittal, K., \&Tayal, D. K. (2014). Automatically incorporating context meaning for query expansion using graph connectivity measures. Progress in Artificiel Intelligence, 2(2-3), 129-139. https://doi.org/10.1007/s13748-014-0041-x

[8] Wikipedia contributors, "DBpedia," Wikipedia, The Free Encyclopedia, 18 Sep. 2019. https://en. wikipedia.org/w/index.php?title=DBpedia\&oldid=916286423. Accessed 03 Nov 2019.

[9] Bouchoucha, A., He, J., \& Nie, J. Y. (2013, October). Diversified query expansion usingconceptnet. In Proceedings of the 22nd ACM international conference on Information \& Knowledge Management (pp. 1861-1864). https://doi.org/10.1145/2505515.2507881

[10] Bendersky, M., Metzler, D., \& Croft, W. B. (2012, February). Effective query formulation with multiple information sources. In Proceedings of the fifth ACM international confer- 
ence on Web search and data mining (pp. 443-452). https://doi.org/10.1145/2124295 .2124349

[11] Yuvarani, M., Iyengar, N. C. S., \&Kannan, A. (2013). Improved concept-based query expansion using Wikipedia. International Journal of Communication Networks and Distributed Systems, 11(1), 26-41. https://doi.org/10.1504/ijends.2013.054833

[12] Dahir, S., El Qadi, A., \&Bennis, H. (2019). Query expansion using Wikidata attributes' values. In ICCWCS 2019: Third International Conference on Computing and Wireless Communication Systems, ICCWCS 2019, April 24-25, 2019, (p. 241). European Alliance for Innovation. https://doi.org/10.4108/eai.24-4-2019.2284070

[13] Min, J. (2017). Utilizing external resources for enriching information retrieval (Doctoral dissertation, Dublin City University).

[14] Keikha, A., Ensan, F., \&Bagheri, E. (2018). Query expansion using pseudo relevance feedback on Wikipedia. Journal of Intelligent Information Systems, 50(3), 455-478. https://doi.org/10.1007/s10844-017-0466-3

[15] Cao, G., Nie, J. Y., Gao, J., \& Robertson, S. (2008, July). Selecting good expansion terms for pseudo-relevance feedback. In Proceedings of the 31st annual international ACM SIGIR conference on Research and development in information retrieval (pp. 243-250). https://doi.org/10.1145/1390334.1390377

[16] Xiong, C., \&Callan, J. (2015, September). Query expansion with freebase. In Proceedings of the 2015 international conference on the theory of information retrieval (pp. 111-120). https://doi.org/10.1145/2808194.2809446

[17] Jeh, G., \&Widom, J. (2002, July). Simrank: a measure of structural-context similarity. In Proceedings of the eighth ACM SIGKDD international conference on Knowledge discovery and data mining (pp. 538-543). https://doi.org/10.1145/775047.775126

[18] Passant, A. (2010, March). Measuring Semantic Distance on Linking Data and Using it for Resources Recommendations. In AAAI spring symposium: linked data meets artificial intelligence (Vol. 77, p. 123).

[19] Alsarem, M. (2013, April). A Generic Approach Based on Linked Data to Enhance Web Information Retrieval and Increase User Satisfaction. In CORIA (pp. 299-304).

[20] Lu, B. (2015). Health Query Expansion Using WordNet and UMLS (Doctoral dissertation, Applied Sciences:).

[21] Hayes, J., \& Gutierrez, C. (2004, November). Bipartite graphs as intermediate model for RDF. In International Semantic Web Conference (pp. 47-61). Springer, Berlin, Heidelberg. https://doi.org/10.1007/978-3-540-30475-3_5

[22] Sun, J., Qu, H., Chakrabarti, D., \&Faloutsos, C. (2005). Relevance search and anomaly detection in bipartite graphs. ACM SIGKDD Explorations Newsletter, 7(2), 48-55. https://doi.org/10.1145/1117454.1117461

[23] Sinha, R., \&Mihalcea, R. (2007, September). Unsupervised graph-based word sense disambiguation using measures of word semantic similarity. In International conference on semantic computing (ICSC 2007) (pp. 363-369). IEEE. https://doi.org/10.1109/icsc.20 $\underline{07.87}$

[24] Keyvanpour, M., \&Serpush, F. (2019). ESLMT: a new clustering method for biomedical document retrieval. Biomedical Engineering/BiomedizinischeTechnik, 64(6), 729-741. https://doi.org/10.1515/bmt-2018-0068

[25] Melethadathil, N., Nair, B., Diwakar, S., \&Heringa, J. (2019). Mining Inter-Relationships in Online Scientific Articles and its Visualization: Natural Language Processing for Systems Biology Modeling. International Journal of Online and Biomedical Engineering, 15(2), 39-59. https://doi.org/10.3991/ijoe.v15i02.9432 
[26] Daoud, D. M., Aoudi, S., \& oudi, M. S. A. (2020). Building a Sentiment Analysis system using automatically generated training Dataset. International Journal of Online and Biomedical Engineering, 16(6), 48-59. https://doi.org/10.1145/3328833.3328874

[27] Navigli, R., \&Lapata, M. (2009). An experimental study of graph connectivity for unsupervised word sense disambiguation. IEEE transactions on pattern analysis and machine intelligence, 32(4), 678-692. https://doi.org/10.1109/tpami.2009.36

[28] Measures. https://trec.nist.gov/pubs/trec10/appendices/measures.pdf. Accessed 03 Nov 2019.

[29] Wikipedia contributors, Evaluation measures (information retrieval). Wikipedia, The Free Encyclopedia. Wikipedia, The Free Encyclopedia, 23 Mar. 2019. Web. 17 Apr. (2019).

[30] Achemoukh, F., \& Ahmed-Ouamer, R. (2018). Integration of User Profile in Search Process according to the Bayesian Approach. International Journal of Recent Contributions from Engineering, Science \& IT (iJES), 6(4), 32-48. https://doi.org/10.3991/ijes.v6i4.9716

\section{Authors}

Sarah Dahir is currently working as Ph.D. at IMAGE Laboratory, SCIAM Team, Graduate School of Technology, Moulay Ismail University of Meknes, Morocco (sarah.dahir2012@gmail.com).

Abderrahim El Qadi is the Professor at ENSAM, Mohammed V University in Rabat, Morocco (abderrahim.elqadi@um5.ac.ma).

Hamid Bennis is the Professor at IMAGE Laboratory, SCIAM Team, Graduate School of Technology, Moulay Ismail University of Meknes, Morocco (hamid.bennis@gmail.com).

Article submitted 2021-03-19. Resubmitted 2021-04-12. Final acceptance 2021-04-12. Final version published as submitted by the authors. 